\title{
Perspective
}

PERSPECTIVE Actualité en histoire de l'art

2| 2007

La Grande-Bretagne/Période moderne

\section{Rubens et les Palazzi di Genova}

\section{Olga Medvedkova}

\section{OpenEdition}

\section{Journals}

Édition électronique

URL : http://journals.openedition.org/perspective/3837

DOI : 10.4000/perspective.3837

ISSN : 2269-7721

Éditeur

Institut national d'histoire de l'art

Édition imprimée

Date de publication : 30 juin 2007

Pagination : 381-386

ISSN : 1777-7852

\section{Référence électronique}

Olga Medvedkova, «Rubens et les Palazzi di Genova », Perspective [En ligne], 2 | 2007, mis en ligne le 31 mars 2018, consulté le 01 octobre 2020. URL : http://journals.openedition.org/perspective/3837 ; DOI : https://doi.org/10.4000/perspective.3837 


\title{
Rubens et les Palazzi di Genova
}

\author{
Olga Medvedkova
}

\section{RÉFÉRENCE}

Herbert W. Rott éd., Rubens Palazzi di Genova. Architectural Drawings and Engravings, Londres, Harvey Miller Publishers, 2002. 2 vol., 575 p., 372 fig. ISBN : 1-872501-33-8 ;

$262,77 €$.

Piet Lombaerde, Krista de Jonge éd., The Reception of P. P. Rubens's Palazzi di Genova

During the 17th Century in Europe. Questions and Problems, (Architectura Moderna

architectural exchanges in Europe 16th - 17th centuries, 1), Turnhout, Brepols, 2002. 262 p., 200 fig. n. et b. ISBN : 978-2-503-51301-0; $86 €$.

1 En 1977, l'année du quatrième centenaire de la naissance de Rubens, dans un numéro spécial du Burlington Magazine, Antony Blunt n'hésitait pas à employer l'expression « Rubens's style in architecture » dans son article "Rubens and Architecture ${ }^{1}$. Il s'agissait moins de l'architecture au sens propre que de la représentation de l'architecture dans les tableaux et les dessins du peintre, notamment dans les dessins qu'il réalisait pour des livres, ou encore ceux pour la Pompa Introitus Ferdinandi ${ }^{2}$.

Paradoxalement, affirmait A. Blunt, dans toutes ces productions, on ne voit que très peu de rapport avec les Palazzi di Genova, l'unique livre d'architecture publié par Rubens. Les créations architecturales de l'artiste étaient en revanche très fortement influencées par les reconstructions de la Rome antique réalisées par les antiquaires humanistes de la fin du $\mathrm{XVI}^{\mathrm{e}}$ siècle, comme celles publiées dans le livre d'antiquités d'Antonio Labacco ${ }^{3}$ ou dans les Cinque libri di architettura de Giovanni Battista Montano (1534-1621 ; l'ouvrage est publié en 1691). Rubens aurait pu avoir rencontré Montano durant son séjour à Rome, puisqu'ils avaient un ami commun - l'antiquaire Fulvio Orsini (1529-1600) - et il avait donc pu consulter ses dessins. Le recours à cette source permettait à A. Blunt de donner une clé pour comprendre le sens de ces décors.

De plus, il remarquait que Rubens avait été le seul grand artiste de son temps à comprendre l'œuvre tardive de Michel-Ange et à l'assimiler de manière libre, et allait 
jusqu'à affirmer que les livres de gravures, publiés par Plantin d'après les dessins de Rubens et remplis de ses fantaisies architecturales, avaient pu influencer Le Bernin et Borromini car ils se trouvaient dans les bibliothèques des ordres religieux auxquels ces architectes avaient été liés. Ainsi, dans son article sur Rubens et l'architecture, A. Blunt ne consacrait que quelques mots aux Palazzi di Genova.

Pendant longtemps, en effet, l'ouvrage ne semblait avoir eu qu'une importance limitée et plutôt locale. L'étude en était menée essentiellement par les spécialistes de l'architecture de Gênes, notamment, dans les années 1920, par Mario Labo. Les planches gravées et les dessins des Palazzi ont été principalement utilisés pour documenter l'histoire de l'architecture à Gênes dans la seconde moitié du XVI ${ }^{e}$ et le début du XVII siècle.

5 Leur publication scientifique semblait poser beaucoup trop de problèmes, aussi bien aux spécialistes de Rubens qu'aux historiens de l'architecture et ce jusqu'à la parution, en 2002, des volumes dus à Herbert W. Rott au sein du prestigieux Corpus Rubenianum fondé par Ludwig Burchard. H.W. Rott semble revenir, en la justifiant et en l'approfondissant, à l'intuition de Jacob Burckhardt. Ce dernier décrivait ce recueil comme l'une des tentatives les plus spectaculaires de la transmission de modèles de l'Italie de la Renaissance vers les pays du Nord.

6 L'ouvrage d'H. W. Rott se compose de deux volumes. Le premier propose une étude historique ainsi que le catalogue de tous les documents graphiques, classés par bâtiment, avec une transcription des notes manuscrites. Il comporte également la reproduction des trois textes imprimés dans le livre : la dédicace à Carlo Grimaldi, la préface de Rubens et l'imprimatur du chanoine anversois Laurent Beyerlinck. Écrits en italien, lingua franca des connaisseurs de l'époque, ces trois textes sont par ailleurs traduits en anglais. Le second volume contient la reproduction de l'ensemble des planches avec, en regard, les dessins à partir desquels les cuivres furent réalisés (les 139 gravures sont réalisées d'après 137 dessins, dont 122 existent encore) et qui se trouvent depuis 1908 dans la collection du Royal Institute of British Architects (RIBA) ${ }^{4}$.

7 Dans sa préface, H.W. Rott justifie la légitimité de ces volumes au sein du Corpus Rubenianum, même si Rubens n'est pas lui-même l'auteur des dessins, car l'ensemble de la publication porte sa marque indélébile. C'est en effet l'unique ouvrage d'architecture (et l'unique ouvrage tout court) publié par le peintre, qui plus est accompagné de son unique texte théorique. Les dessins pour l'édition furent rassemblés par Rubens luimême : ils font donc partie intégrante de sa collection.

8 L'ensemble de sources rassemblées par H. W. Rott autour des Palazzi di Genova fait de son ouvrage un outil exceptionnel et sans doute jusqu'à aujourd'hui l'unique exemple de publication de cette ampleur et de cette qualité consacrée à un seul livre d'architecture. La qualité de l'essai historique y est particulièrement remarquable. L'auteur fournit d'abord les clés nécessaires à la bonne "lecture » de l'ouvrage et à la compréhension de son type et de sa structure. Il reconstitue ensuite l'histoire de son édition.

9 L'ouvrage, paru en printemps 1622 (le privilège datait de janvier 1620), réunissait 72 planches gravées par Nicolaes Ryckemans (graveur des œuvres picturales de Rubens) qui représentaient douze villas et palais de Gênes, tous construits à la fin du XVI siècle avec, comme point central, les créations de l'architecte Galeazzo Alessi. Dans sa préface, l'artiste exprimait une vision humaniste de l'histoire de l'architecture qui 
connut une renaissance par le retour à l'antique après le déclin du Moyen Âge. Ces nouvelles idées architecturales avaient déjà été introduites dans son pays, mais seulement dans les édifices religieux, et le but de Rubens était donc de les promouvoir dans le domaine de l'habitat privé. Pour cela, il offrait au public une version gravée de la collection de dessins qu'il avait achetés durant son séjour en Italie (1600-1608). Il fournissait ainsi des modèles de constructions privées fondés sur les règles de la "bonne architecture ", prestigieuse en même temps qu'économique. Il parlait ensuite de la commodité, comme de la principale notion de l'architecture domestique.

Cette monographie in folio consacrée à l'architecture privée et moderne d'une ville n'avait pas de précédents car une documentation aussi riche et variée était réservée auparavant aux constructions princières ou aux édifices antiques. Il existait avant Rubens des recueils de gravures de bâtiments privés : ceux de Serlio, d'Androuet du Cerceau, ou encore, plus proches, ceux du second livre de Palladio. Mais il n'y avait jamais eu de publication de maisons existantes (et étrangères!) proposées comme modèles pour un autre pays. Plus que des livres de modèles, Rubens s'inspirait sans doute d'éditions comme le speculum romanae magnificentiae d'Antonio Lafreri et communiquait le prestige de l'Antiquité à l'architecture moderne fondée sur les principes de l'antique.

11 Les planches ne furent dotées d'aucun texte d'explication. À chaque bâtiment Rubens consacra jusqu'à neuf gravures de grand format, montrant des plans, des élévations et des coupes longitudinales et transversales. Les mesures et les désignations des pièces étaient portées sur toutes les gravures, et cela non seulement dans les parties principales, mais également dans les parties destinées au service. Le second ensemble de 67 planches, très différentes des 72 premières par leur style simplifié, représentait les 23 palais construits entre 1540 et 1620 avec le nom de leurs propriétaires, ainsi que plusieurs églises de Gênes de la même époque. Il fut publié entre 1622 et 1642.

12 Après la mort de Rubens, le livre fut réédité quatre fois à partir des cuivres originaux (en 1652, 1663, 1708 et en 1755). Dans la première réédition, Jakob Meursius introduisit la division entre le premier groupe nommé Palazzi Antichi et le second, nommé Palazzi Moderni. En réalité, les palais de la première partie étaient parfois plus récents que ceux de la seconde. Dans l'édition d'Amsterdam de 1755, les textes furent traduits en français et on ajouta la Vie de Rubens de Jean Baptiste Descamps.

13 L'analyse de H.W. Rott montre en outre à quel point l'histoire de la théorie architecturale peut être essentielle et fructueuse quand elle est nourrie de l'histoire sociale ainsi que de l'histoire de la pensée politique. Depuis la naissance du mouvement humaniste, les architectes et les philosophes pensaient la Cité en commun, notamment autour du projet vitruvien, et le livre d'architecture se trouvait au cœur de cette entreprise $e^{5}$. Or les palais de Gênes étaient presque autant des résidences privées que des édifices civils. L'ensemble de palais publiés par Rubens est en effet marqué non seulement par une unité chronologique (1540-1620) et topographique (ils s'élèvent sur la Strada Nuova et sur la Via Balbi), mais également par une grande unité typologique et sociale. Ils furent commandités surtout par les huit grandes familles de la nobiltà vecchia qui faisaient aussi construire alors les principales églises de la ville. Comme la République ne possédait pas à l'époque de vrai centre représentatif pour les grandes occasions et les cérémonies, ils constituaient le décor dans lequel elle se présentait au monde, servant d'expression architecturale parfaite pour le système oligarchique de 
Gênes. Rubens présentait cette réalité comme idéale et proclamait la possibilité de son transfert, y compris dans d'autres conditions géographiques et politiques.

Les huit grandes familles correspondaient de plus aux relations génoises de Rubens qui pourtant ne mentionnait pas les noms des propriétaires dans la première partie de son ouvrage, sans doute pour renforcer l'effet d'un livre de modèles et pour faciliter leur adaptation dans le Nord. En tout cas, cet anonymat des maisons particulières augmentait l'idée du «corps » unique d'une ville-république. Ainsi, sous l'apparence d'une édition consacrée à Gênes, Rubens offrait-il peut-être une réflexion en image sur une ville idéale.

H. W. Rott essaie ensuite de comprendre le choix esthétique de Rubens à partir de ces réalités politiques et sociales. L'aspect des premiers palais de Gênes devait beaucoup à l'architecte Galeazzo Alessi (1512-1572) qui fut formé dans le cercle romain d'Antonio da Sangallo le Jeune (1483-1546). La ressemblance des autres palais avec ceux projetés par Alessi était étonnante. Elle était principalement le fait des commanditaires : selon les archives, les propriétaires, qui faisaient appel aux mêmes architectes et au même groupe de constructeurs et de décorateurs, commandaient jusqu'aux détails de décors de leurs palais en demandant l'imitation des exemples voisins. Ainsi, les palais de Gênes fonctionnaient eux-mêmes, les uns par rapport aux autres, comme des modèles, et ce dès le moment de leur création.

L'analyse de ce fonctionnement constitue les pages les plus passionnantes de l'ouvrage. Elle permet de comprendre le contenu réel de la «formule» de la ville utopique, composée de maisons privées semblables. Aussi, selon H. W. Rott, les dessins de Rubens étaient-ils réalisés comme un recueil d'exempla servant lors des discussions entre architectes, artisans et commanditaires, pour être collectionnés par les connaisseurs appartenant au patriciat génois - fier et conscient de la réussite de son «projet urbain »- ou pour remplir les collections étrangères. Puisque les Génois voulaient posséder ou faire circuler les images de leurs palais pour augmenter leur prestige, on serait tenté de supposer que cette collection avait été préparée comme un projet de publication que Rubens ne fit que réaliser.

Il s'agit, enfin, de comprendre les raisons pour lesquelles Rubens réalisa cette publication. Et c'est là qu'H. W. Rott rejoint les propos d'A. Blunt. Certes, depuis qu'il s'était installé à Anvers, Rubens était sensible à l'architecture. Comme peintre de l'église des Jésuites à Anvers, il influença sinon participa directement à l'élaboration de son décor architectural. Il construisait en même temps sa propre maison et achetait de nombreux livres d'architecture pour sa bibliothèque. Et pourtant, bien qu'il présentât son livre au public comme un livre de modèles pour les architectes d'Anvers, lui-même ne les utilisa jamais, ni dans ses constructions ni dans ses architectures dessinées ou gravées. De même, dans le contexte de sa collection (qui n'est pas un cabinet de curiosité encyclopédique mais une collection «de travail» d'un artiste humaniste), essentiellement orientée vers l'Antiquité, cet ensemble de dessins fait figure d'un corps étranger.

Les raisons de la publication des Palazzi deviennent pourtant plus facilement envisageables quand l'auteur la resitue par rapport à la personnalité de Rubens, ainsi que dans le contexte politique. Les Palazzi seraient alors intimement liés au désir que Rubens avait d'apparaître comme un expert dans le domaine de l'architecture. Avec la collection de dessins qu'il possédait, il pouvait réaliser un livre d'architecture à peu de frais, avec l'efficacité qu'il recherchait dans toutes ses entreprises. La publication 
pouvait également toucher la monarchie espagnole, très liée à Gênes (les troupes espagnoles faisaient halte à Gênes sur la route des Pays-Bas; elles étaient financées principalement par les banquiers génois; leur payement s'effectuait partiellement par les branches des banques génoises installées à Anvers).

19 Ainsi l'auteur parvient à éclaircir plusieurs problèmes liés aux Palazzi. La solution du paradoxe d'A. Blunt devient envisageable. La source universelle et absolue se trouvait pour Rubens dans l'Antiquité romaine : elle seule - directement ou par le biais de ses grands interprètes, comme l'était Michel-Ange - pouvait nourrir un doctus artifex. Tout projet moderne devait se fonder sur les modèles antiques. L'utopie réalisée de Gênes offrait un paradigme de ce type d'entreprise et devait servir d'exemple aux architectes du Nord. Ces derniers, peu savants, avaient besoin d'un livre de modèles. En leur offrant celui-ci, Rubens pouvait prétendre à les «diriger». Quant à ses propres " architectures ", il se réservait un droit exclusif de recourir à la source, l'Antiquité, réservée aux savants.

20 Nombre de problèmes évoqués par $\mathrm{H}$. W. Rott sont repris dans un ouvrage collectif, actes d'un colloque international, qui constitue le premier volume de la collection Architectura Moderna: Architectural Exchanges in Europe, $16^{\text {th }}-17^{\text {th }}$ Centuries, dirigée par Krista De Jonge et Piet Lombaerde.

21 Les trois premiers articles approfondissent les recherches liées à la production de l'ouvrage. L'article de Frans Baudouin est, par exemple, consacré à l'évolution du statut $\mathrm{du}$ « peintre-architecte» et permet de mieux comprendre dans quelle mesure Rubens pouvait prétendre à pratiquer le «métier » d'architecte. Clara Altavista, à travers une analyse quasi philologique des dessins et planches par rapport aux bâtiments génois, étudie comment la réalité architecturale de Gênes avait été triée, sélectionnée, classée et finalement codifiée en dessin et en gravure pour l'édition de Rubens.

Dans un article important, Piet Lombaerde développe la place de cette édition dans le contexte de la théorie et de l'édition architecturale du xvir siècle. Il analyse le principal propos de Rubens, à savoir l'introduction à Anvers de la "bonne " architecture moderne fondée sur les règles classiques, du nouveau type de la maison urbaine (en comparaison avec la maison classique et la maison rustique qui figurent chez Serlio), adapté à « la nouvelle philosophie urbaine ». Cette maison gardait son indépendance et en même temps participait à l'unité de la ville. Sa caractéristique principale était dans le rapport entre la sala, la loggia et le cortile. La formule en fut : « La forma di cubo solido col salone in mezzo ". Ce salon au milieu, ainsi que d'autres pièces de grandes dimensions, appelait le décor pictural, ce qui pouvait fournir à l'atelier de Rubens de nouvelles commandes. L'artiste rêvait sans doute d'une nouvelle ville d'Anvers habitée par des hommes riches et amateurs de peinture, avec des maisons aux pièces spacieuses et des églises aux grands murs vides, remplies de ses peintures.

Pour situer le livre dans l'ensemble de l'édition architecturale, Piet Lombaerde évoque les idées d'Alberti et de Serlio. Il compare Rubens avec Le Muet, Jombert, Louis Savot, Alessandro Francini et Joseph Furttenbach. L'exemple le plus proche serait selon lui le livre de Salomon de Bray Architectura Moderna (Amsterdam, 1631). Il souligne ensuite le fondement théorique de Rubens dans le domaine de l'architecture et consacre une analyse très fouillée à son interprétation de Vitruve.

Les autres contributions explorent les problèmes de la circulation des Palazzi à travers l'Europe. Konrad Ottenheym analyse l'influence de l'ouvrage sur l'architecture de l'ensemble des Pays-Bas, et Piet Lombaerde sur celle des Pays-Bas Méridionaux. John 
Newman étudie la présence de l'ouvrage à Londres et sa réception à travers les dessins et les notes de John Webb, élève et héritier d'Inigo Johns, et les écrits d'un architecte amateur, Roger Pratt. L'étude de Charles Hind apporte de nouveaux éléments pour l'histoire de la collection des dessins de Rubens qui se trouve au RIBA. Ulrich Schütte traite de l'influence des Palazzi di Genova sur la nouvelle orientation donnée à l'architecture allemande par Joseph Furttenbach l'Ancien, par les traités d'architectures et d'urbanisme que publie ce marchand d'Ulm. Enfin, Joris Snaet replace l'ouvrage de Rubens dans le contexte de la construction des deux églises jésuites d'Anvers et de Bruxelles. Ces actes sont complétés par la reproduction des planches de l'ouvrage conservé dans une bibliothèque privée anversoise.

Claude Mignot retrace la réception des Palazzi di Genova en France au XVII ${ }^{\mathrm{e}}$ siècle. Il mentionne les envois du recueil auxquels Rubens procéda lui-même, la présence du traité dans les bibliothèques des architectes français ou dans celles des amateurs. Il poursuit par l'analyse des œuvres architecturales. Les quelques exemples cités, dont les hôtels de Louis Le Vau, permettent d'affirmer que cette influence fut «limitée, plus bibliophilique qu'architecturale ». C. Mignot s'attache ensuite à l'édition française de 1755 dans le contexte du retour aux modèles italiens, antiques et modernes. Il souligne que Fontaine possédait un exemplaire des Palazzi dans l'édition francisée de 1755 (celui qui se trouve à la bibliothèque de l'INHA - collections Jacques Doucet) ${ }^{6}$. Et il est en effet difficile de ne pas voir dans l'édition de Rubens l'un des prototypes du livre de Percier et Fontaine, consacré aux palais de Rome, de même que de celui de Pierre Gauthier qui, en 1818, publia sa propre version des palais de Gênes.

D'une façon générale, comme le souligne Piet Lombaerde, la circulation du livre et son influence sur l'architecture doivent être considérées comme deux sujets séparés. Le second point « repose essentiellement sur des comparaisons iconographiques qui ne sont pas soutenues par les documents d'archives ou par les dessins. Pour cette raison, elles restent purement spéculatives et hypothétiques» (p.99). En revanche la circulation du livre repose sur des documents.

Il faudrait sans doute ajouter à l'étude des inventaires des bibliothèques un recensement des différents exemplaires des Palazzi conservés. Chacun recèle en effet des informations significatives, comme celui de la première édition qui se trouve à la bibliothèque de l'INHA ${ }^{7}$. Malgré le fait que Rubens y prêchait contre l'architecture "barbare et gothique ", il fut relié avec trois des quatre célèbres gravures d'Isaac Brun représentant la cathédrale de Strasbourg. La collection des planches de Rubens fut donc perçue par un collectionneur inconnu du XviI ${ }^{\mathrm{e}}$ siècle comme celle des "vues" topographiques de la ville de Gênes plutôt que comme un ensemble de modèles de la «bonne architecture». En revanche, la présence de l'ouvrage dans la collection de Pierre le Grand (Bibliothèque de l'Académie des Sciences, Saint-Pétersbourg), qui cherchait les « modèles à imiter " pour la nouvelle ville de Saint-Pétersbourg, témoigne d'une parfaite « figure de transfert».

Un ensemble d'études de cette qualité et de cette ampleur ne peut que faire rêver un historien de la théorie architecturale française. Et cela d'autant plus que c'est en France, à partir surtout du milieu du $\mathrm{XvII}^{\mathrm{e}}$ siècle, que furent publiés des traités d'architecture de toute première importance. Leurs multiples rééditions et traductions inondèrent ensuite l'Europe et déterminèrent les principaux courants de la pensée architecturale de la période moderne. Il suffit, pour s'en convaincre, de penser au Parallèle de l'architecture antique avec la moderne de Fréart de Chambray (1650) ou de la 
traduction du De Architectura de Vitruve par Claude Perrault (1674). La genèse et la fortune de tels ouvrages pourraient donner lieu à des études non moins fructueuses que celles dont nous venons de parler.

\section{NOTES}

1. Antony Blunt, «Rubens and Architecture », dans The Burlington Magazine, CXIX, sept. 1977, p. 609-621.

2. Pompa triumphalis introitus Ferdinandi Austriaci Hispaniarum infantis, \& c. in urbem Antveroiam Ferdinandi Austriaci..., Anvers, 1636.

3. Libro d'Antonio Labacco appartenente a l'architettvra nel qual si figurano alcune notabili antiquita di Roma, Rome, 1557 ; voir Michaël Jaffe, «Rubens and Optic ", dans Journal of the Warburg and Courtauld Institutes, XXXIV, 1971 ; voir également son Rubens and Italy, Oxford, 1977.

4. Ils ont été publiés dans Jill Lever éd., Catalogue of the Drawings Collection of the Royal Institute of British Architects, Farnborough, 1976.

5. Voir par exemple Ingrid Rowland, The culture of the High Renaissance. Ancients and moderns in sixteenth-century Rome, Cambridge, 1998.

6. L'édition conservée sous la cote Fol C 211 : Architecture italienne, contenant les plans et élévations des plus beaux palais et édifices de la ville de Gènes levé et dessiné par le célèbre P. P. Rubbens, troisième édition augmentée d'un abrégé de la vie de l'auteur, à Amsterdam et à Leipzig, chez Arkstee \& Mercus, 1755, avec une reliure du XVIII ${ }^{\mathrm{e}}$ siècle, plein veau marron jaspé, dos à six nerfs, et un titre collé sur une étiquette au dos «ARCHIT DE RUBBEN »; contient un ex-libris sur la page de garde : tampon de forme ronde à l'encre noire «P.F. L. Fontaine » avec, ajouté au crayon, " architecte », « $\mathrm{n}^{\circ} 327$ » et de multiples notes manuscrites au crayon.

7. Collections Jacques-Doucet, Fol C 23, avec une reliure vélin, début XVII ${ }^{\mathrm{e}}$ siècle.

\section{INDEX}

Mots-clés : architecture, peinture palais, ville, dessin, peintre-architecte Index géographique : Italie, Gênes, Rome

Keywords : architecture, painting, palace, city, drawing, painter-architect Index chronologique : 1600

\section{AUTEURS}

\section{OLGA MEDVEDKOVA}

INHA 
